\title{
Radio Interferometric Planet Search II: Constraints on sub-Jupiter-Mass Companions to GJ 896A
}

\author{
Geoffrey C. Bower ${ }^{1}$, Alberto Bolatto ${ }^{1,2}$, Eric B. Ford ${ }^{1,3}$, Adam Fries ${ }^{1}$, Paul Kalas ${ }^{1}$, Karol \\ Sanchez $^{1}$, Phoebe Sanderbeck ${ }^{4}$, Vincent Viscomi ${ }^{1}$ \\ gbower@astro.berkeley.edu
}

\begin{abstract}
We present results from the Radio Interferometric Planet (RIPL) search for companions to the nearby star GJ 896A. We present 11 observations over 4.9 years. Fitting astrometric parameters to the data reveals a residual with peak-to-peak amplitude of $\sim 3$ mas in right ascension. This residual is well-fit by an acceleration term of $0.458 \pm 0.032$ mas $/ \mathrm{y}^{2}$. The parallax is fit to an accuracy of 0.2 mas and the proper motion terms are fit to accuracies of $0.01 \mathrm{mas} / \mathrm{y}$. After fitting astrometric and acceleration terms residuals are 0.26 mas in each coordinate, demonstrating that stellar jitter does not limit the ability to carry out radio astrometric planet detection and characterization. The acceleration term originates in part from the companion GJ 896B but the amplitude of the acceleration in declination is not accurately predicted by the orbital model. The acceleration sets a mass upper limit of $0.15 M_{J}$ at a semi-major axis of $2 \mathrm{AU}$ for a planetary companion to GJ 896A. For semi-major axes between 0.3 and 2 AU upper limits are determined by the maximum angular separation; the upper limits scale from the minimum value in proportion to the inverse of the radius. Upper limits at larger radii are set by the acceleration and scale as the radius squared. An improved solution for the stellar binary system could improve the exoplanet mass sensitivity by an order of magnitude.
\end{abstract}

Subject headings: astrometry, stars: activity, stars: early-type, stars: planetary systems, radio continuum: stars

\footnotetext{
${ }^{1}$ Astronomy Department \& Radio Astronomy Laboratory,University of California, Berkeley, CA 94720

${ }^{2}$ Department of Astronomy, University of Maryland, College Park, MD, 20742-2421

${ }^{3}$ Astronomy Department, University of Florida, Gainesville, FL 32611-2055

${ }^{4}$ Department of Physics, Brandeis University, Abelson-Bass-Yalem 107, MS 057, 415 South Street, Waltham, MA 02453
} 


\section{Introduction}

The study of extrasolar planets provides an important link between the study of star formation, the study of our own solar system, and the search for extraterrestrial life. Radial velocity searches (Butler et al. 2006), transit searches with Kepler (Borucki et al. 2011), and microlensing searches (Gaudi et al. 2008) have surveyed significant components of the exoplanet mass-separation parameter space exposing a wide variety of phenomenology.

Despite their abundance, low mass stars have been less well-characterized for a number of reasons. In particular, low mass stars are often too faint or too strongly variable for high precision radial velocity and transit searches. Microlensing searches have identified companions to low mass stars (Bennett et al. 2008; Beaulieu et al. 2006; Gould et al. 2006), which suggest that such companions may be very common. On the other hand, radial velocity searches suggest very low planet occurrence rates for close-in companions (Endl et al. 2006; Johnson et al. 2007). The frequency and distribution of companions to low mass stars can be an important diagnostic of methods for planet formation (Laughlin et al. 2004; Ida \& Lin 2005; Boss 2006; Kennedy et al. 2007).

Astrometry can be a powerful tool for detection and characterization of nearby, low-mass planetary systems. Muterspaugh et al. (2010) demonstrate that optical astrometric measurements with the Palomar Testbed Interferometer are capable of achieving sub-milliarcsecond accuracy in the separation between close binaries. They summarize the known properties of sub-stellar companions to low mass stars in binary systems and conclude that giant planet companions in such systems are common. The Carnegie Astrometric Planet Search Program (Boss et al. 2009) has also obtained sub-milliarcsecond astrometry on the low mass star NLTT 48256 over 2 years of observations; this program will ultimately survey 100 nearby M, L, and T dwarfs.

The Very Long Baseline Array (VLBA) can routinely achieve an astrometric accuracy of $\sim 100$ $\mu$ as in a single epoch at frequencies $\lesssim 10 \mathrm{GHz}$ and $\sim 20 \mu$ as at higher frequencies. The VLBA is capable of accuracies as high as $8 \mu$ as under favorable circumstances (Fomalont \& Kopeikin 2003). To use this technique the target source must have a sufficiently high brightness temperature to be detected by a high resolution radio interferometer. For the VLBA, brightness temperatures must be $T_{b} \gtrsim 10^{7} \mathrm{~K}$, requiring nonthermal emission. Thus, the systems which can be studied are limited to the most active stars. VLBA astrometry has been important for a wide range of stellar measurements including parallax (e.g., Sandstrom et al. 2007; Menten et al. 2007; Loinard et al. 2007; Torres et al. 2009), galactic structure (Xu et al. 2006, 2011; Brunthaler et al. 2011), binary orbit characterization (Guirado et al. 2006), and the radio/optical reference-frame link (Lestrade et al. 1999). Recently, Forbrich \& Berger (2009) detected a brown dwarf with the VLBA and explored

the possibilities of companion detection. Bower et al. (2007) reviewed the prospects for future radio astrometric surveys with respect to other methods.

RIPL, the Radio Interferometric Planet Search, is a 4-year program using the VLBA and the 100m Green Bank Telescope (GBT) to search for the astrometic signatures of massive planets around nearby $(D<10 \mathrm{pc})$, low-mass (M dwarf) stars. We are observing a sample of 30 stars a total 
of 12 times over a 4-year period with the expectation of achieving $\sim 0.1$ milliarcsecond astrometric accuracy per epoch, and ultimately having the sensitivity to detect Jupiter-mass planets at 1 AU. This astrometric search is an important complement to radial velocity, transit, microlensing, and optical astrometric searches for planets on account of greater sensitivity to 1) low mass stars, 2) long-period planets, 3) active stars, and, 4) planets that may be imaged with extreme adaptive optics. Many RIPL stars are in binary systems, which may have higher planetary fractions.

In Paper I (Bower et al. 2009), we used Very Large Array (VLA) observations to identify the sample of stars appropriate for RIPL and we used the VLBA to characterize the detectability and short-term $(\lesssim 10$ days) stability of the astrometric images of these stars. The short-term stability addresses a significant concern of radio astrometry that stellar activity will lead to large astrometric errors. The absence of a significant difference in the historical optical proper motions and the radio proper motion from this RIPL precursor survey permitted us to exclude brown dwarf companions to the four stars observed. RIPL observing began in October 2007 and is anticipated to end in late 2011.

In this paper, we present initial results from RIPL for the star GJ 896A. This star was also observed as part of the precursor survey in paper I. Together these observations demonstrate astrometric stability over a 4.9 year span. GJ 896A (EQ Peg A) is a variable star in a multiple system with GJ 896 B, C, and D. GJ 896B is its closest companion; the orbit has been characterized, but it is not well constrained (Heintz 1984). GJ 896 A and B have spectral types M3.5 and M4.5. These two stars have been detected previously in the radio and seen to have point-like structure and strong radio variability (Pallavicini et al. 1985; Jackson et al. 1989; Benz et al. 1995; Gagne et al. 1998). In $\S 2$, we describe our observations, analysis, and results. In $\S 3$, we present our astrometric analysis of the data. In $\S 4$, we examine the constraints that we can place on companions to GJ 896A. In $\S 5$, we summarize our results.

\section{Observations, Data Analysis, and Results}

Observations of GJ 896A were obtained as part of the RIPL survey. RIPL observations spanned October 2007 through June 2011, and are ongoing. In each epoch, we observed two stars over a span of 8 hours. Fast switching with a phase reference calibrator and secondary (and sometimes tertiary)

astrometric calibrators reduced total observing time on each star to approximately two hours. The total span from start to end of observation for a particular star within an epoch varied, depending on the relative coordinates of the stars and the allocated time slot. In some instances, the stars were observed sequentially in four hour blocks. In other instances, the stars were interleaved and the span was over the full eight hours.

Observations typically included all ten antennas of the Very Long Baseline Array as well as the 100 meter Green Bank telescope. Data were recorded at a rate of $512 \mathrm{Mbps}$ in dual circular polarization mode with observing bands at $8.4 \mathrm{GHz}$ using the standard VLBA mode v4cm-512- 
8-2. More precisely, this mode corresponds to four dual-polarization frequency bands centered at $8384.49,8400.49,8416.49$, and $8432.49 \mathrm{MHz}$, each sampled at 32 Msamples/second with 2 bit sampling. Occasional observations of fringe calibrators were interspersed throughout each epoch. The switching cycle included repeated observations of 1 minute on the phase reference calibrator followed by 3 minutes on the star. Every 30 minutes short duration observations of the secondary and tertiary calibrators phase referenced to the primary calibrator were obtained.

Data were reduced with an AIPS (Greisen 2003) pipeline that performed standard calibrations of amplitude and phase. Corrections for the atmospheric total electron content (TEC) and postcorrelation updates to the Earth orientation parameters (EOP) were also included. All sources were imaged and deconcolved using the CLEAN algorithm. The typical synthesized beam for GJ 896 A was $3.0 \times 1.1$ mas, oriented North-South. We fit Gaussian models to all sources using the AIPS task JMFIT.

In the case of GJ 896A, the primary phase reference calibrator is J2328+1929, which has an ICRF2 position that is accurate to 1.84 mas $\times 1.58$ mas 1 . The assumed position for J2328+1929 is $\left(23^{h} 28^{m} 24.874773^{s}, 19^{\circ} 29^{\prime} 58.03010^{\prime \prime}\right)$; these updated values are slightly offset from the assumed position of paper I by $(0.254,0.059)$ mas in right ascension and declination, respectively. All positions for other sources are relative to this position. The secondary calibrator is J2334+2010. No tertiary calibrator was used. We apply a correction for tropospheric phase gradients on the astrometric position of the star by removing variations in the position of the secondary calibrator. This correction is applied on an epoch-by-epoch basis. A more sophisticated approach using the AIPS task ATMCA can make corrections on shorter timescales. The magnitude of corrections to GJ $896 \mathrm{~A}$ are $\sim 0.1$ mas. This approach may overestimate trophospheric errors slightly given the greater distance and higher declination of J2334+2010 from the primary calibrator. The close spacing and nearly linear arrangement of the star and calibrators, however, make this a good calibration set (Fig. 1). Typically, 3C 454.3 was observed as the fringe calibrator. As of June 2011, we have 8 good epochs for GJ 896A.

Both calibrators are dominated by a strong point source and show only weak evidence for non-point-like structure. Images of the calibrators are included in Paper I. The primary calibrator, J2328+1929, has a typical flux density of $60 \mathrm{mJy}$; the secondary calibrator, J2334+2010, has a typical flux density of $20 \mathrm{mJy}$. In Table 1, we summarize flux densities and positions for the secondary calibrator as a function of epoch; we also include the mean value from the three epochs of paper I. The positions for J2334+2010 have an rms variation of $(0.11,0.09)$ mas and a mean offset $(0.06,0.22)$ mas from the astrometric position obtained in paper I. That mean offset is within the error bars of the original measurement. These measures for the secondary calibrator provide an estimate of the astrometric accuracy of the experiment.

We summarize positions and flux densities for GJ 896A in Table 2 as a function of epoch. We

${ }^{1}$ http://gemini.gsfc.nasa.gov/solutions/2010a/2010a.html 
also include measurements from Paper I. GJ 896A is strongly variable: flux densities vary from 0.140 to $9.512 \mathrm{mJy}$ during the course of the experiment. The images of GJ 896A are predominantly point-like, as found in Paper I. The smaller errors on the flux density and the positions are reflective of the increased sensitivity and $(u, v)$ coverage of RIPL over the precursor experiment due to the doubling of the observing time, doubling of the bit rate, and addition of the GBT to the array. The statistical errors are significantly less than the systematic errors associated with the astrometry. For the analysis discussed below, we add in quadrature an error of 0.2 mas to each coordinate as an estimate of the systematic error.

\section{Astrometric Fitting}

In addition to the eight epochs from RIPL, we obtained three epochs in the RIPL precursor survey over three days in March 2006. We consider the data from all 11 epochs in our astrometric fitting here. The data span 4.9 years.

GJ 896A was not originally part of the Hipparcos catalog but it is included in the reanalysis of the Hipparcos data (van Leeuwen 2007). We summarize the known astrometric parameters in Table 3. GJ 896A is also known to be in a double system with GJ 896B; parameters are determined from $\sim 40$ years of optical observations (Table 4, Heintz 1984; Mason et al. 2001). Since, the baseline for this orbit determination is $\sim 0.1$ times the estimated period, the orbital parameters are poorly determined. This is reflected in the binary orbit grade of 5 ("indeterminate") on a scale of 1 to 5 .

In Table 3, we show the nominal astrometric data available from optical sources as well as bestfit solutions based on RIPL data. We performed two kinds of fits: one with just the astrometric parameters, and the other with astrometric parameters and an acceleration term. Figures 2, 3, and 4 show the astrometric data, the models, and the residuals for the cases of the initial model, the astrometric model, and the astrometric plus acceleration model, respectively. The astrometric plus acceleration model that we fit to our data $\left(\alpha\left(t_{i}\right), \delta\left(t_{i}\right)\right)$ at epoch $t_{i}$ is

$$
\begin{aligned}
\alpha\left(t_{i}\right) & =\alpha_{0}+\mu_{\alpha}\left(t_{i}-t_{0}\right)+\pi_{\alpha}\left(\alpha, \delta, t_{i}\right)+a_{\alpha}\left(t_{i}-t_{0}^{\alpha}\right)^{2}, \\
\delta\left(t_{i}\right) & =\delta_{0}+\mu_{\delta}\left(t_{i}-t_{0}\right)+\pi_{\delta}\left(\alpha, \delta, t_{i}\right)+a_{\delta}\left(t_{i}-t_{0}^{\delta}\right)^{2}
\end{aligned}
$$

where $\left(\alpha_{0}, \delta_{0}\right)$ is the equinox J2000 position at time $t_{0}$, the epoch $2000.0,\left(\mu_{\alpha}, \mu_{\delta}\right)$ is the proper motion, $\left(\pi_{\alpha}, \pi_{\delta}\right)$ are the projections of the parallax $\pi$ onto the two coordinates, $\left(a_{\alpha}, a_{\delta}\right)$ is the local acceleration, and $t_{0}^{\alpha}$ and $t_{0}^{\delta}$ are zero-points for the acceleration in each coordinate. The initial model clearly shows a substantial error in $\mu_{\alpha}$ and a $\sim 1$ mas error in $\pi$. Fitting the astrometric parameters $\left(\alpha_{0}, \delta_{0}, \mu_{\alpha}, \mu_{\delta}, \pi\right)$ substantially improves the quality of the fit but leaves a parabolic residual in $\alpha$. An acceleration term reduces the rms in $\alpha$ from 1.2 to 0.26 mas. The residual in declination is improved from 0.33 to 0.26 mas through the addition of an acceleration term. The astrometric fit alone with 22 measurements and 5 parameters producing 17 degrees of freedom results in $\chi^{2}=338$. 
The acceleration term significantly reduces $\chi^{2}$ to 29.7 with 9 parameters producing 13 degrees of freedom. The reduced $\chi_{\nu}^{2}=2.3$ in this final fit.

We estimate errors in individual parameters by mapping the $\chi^{2}$ surface as a function of each parameter. For the purely astrometric case, the $\chi^{2}$ curves are parabolic. We plot the $\chi^{2}$ curves for the astrometric and acceleration terms in Figure 5. These curves are also parabolic and symmetric about their best-fit values. We estimate the $1 \sigma$ errors from these curves. The astrometric parameter estimates are significantly more accurate when the acceleration terms are included. We do not estimate the full covariance matrix of the parameter estimates due to the computational cost of exploring a 9-dimensional space. We did explore the covariance between the parallax and the right ascension acceleration term, two parameters that one might expect to be strongly linked. We find that the $\chi^{2}$ surface in this two-dimensional domain is well-characterized by a paraboloidal surface with axes that are misaligned from the principle axes by less than 30 degrees; that is, the parameters are essentially independent.

The estimated values for the astrometric parameters are determined one to two orders of magnitude more accurately than the Hipparcos values. The values for $\alpha_{0}, \delta_{0}, \pi$, and $\mu_{\delta}$ are in agreement with the Hipparcos values. The proper motion in right ascension, $\mu_{\alpha}$, however, differs by $16.5 \pm 1.4 \mathrm{mas} / \mathrm{y}$. Over the time baseline between the Hipparcos epoch (1991.25) and the mean RIPL epoch (2008.75), this implies an acceleration of $0.94 \pm 0.08 \mathrm{mas} / \mathrm{y}^{2}$. This has the same sign and a comparable magnitude to the acceleration term measured from the RIPL data alone. The agreement in $\mu_{\delta}$ implies an upper limit of $0.06 \mathrm{mas} / \mathrm{y}^{2}$ over this interval.

Motion of the star during the observing epoch can be an important effect, especially if the flux density is variable on short timescales. For GJ 896A, the median absolute value of motions from astrometric parameters are $(0.10,0.048)$ mas/hour in the two coordinates, respectively, and are always lower than $(0.14,0.065)$ mas/hour. This can lead to smearing out of the source and reduction of the peak flux density. If the source flux density is strongly variable during the observation, then the mean time of the epoch may not correspond to the time of the astrometric detection, leading to errors be as large as $(0.6,0.3)$ mas. The small residuals we obtain from fitting indicate that this effect is not likely to be important in most epochs. Future reductions of this data will attempt to determine any light curve variability and its effect on the astrometry and/or include corrections to the visibilities for the assumed astrometric motion of the star. In high signal to noise ratio cases, we may be able to simultaneously fit a position and velocity within a given epoch.

\section{Constraints on Companions to GJ 896A}

The best fit accelerations for GJ 896A are $(0.458,0.087) \mathrm{mas} / \mathrm{y}^{2}$. We compare this to the known parameters of the binary system. We plot relative separation, velocity, and acceleration determined from the binary parameters (Figure 6). Note that the definition of the orbit gives the position of $\mathrm{B}$ relative to $\mathrm{A}$. Thus, the sign of velocity and acceleration terms is inverted relative 
to our measurement. If the stars have equal mass, then the motion relative to the center of mass will be half of the value plotted. The mean calculated accelerations for A relative to B over our observing epochs is $(0.3 \pm 0.1,3.1 \pm 0.6) \mathrm{mas} / \mathrm{y}^{2}$. The calculated acceleration term in $\alpha$ agrees reasonably well with the measured value and is comparable to the mean acceleration over the entire orbit $\approx 2 \pi r / P^{2}=0.3 \mathrm{mas} / \mathrm{y}^{2}$. The calculated acceleration term $a_{\delta}$ does not agree at all with the measured value. The RIPL measurements occur near the predicted periastron, and, therefore, a maximum in the value of $a_{\delta}$. Our results clearly indicate a significant error in the model for this binary system and appear to indicate that the period may be longer than 359 years, or that the eccentricity or time of periastron have not been properly estimated.

The acceleration $a$ allows us to a place a limit on the stellar companion mass, $M_{B}$, using

$$
\left(\frac{M_{B}}{M_{J}}\right)=\left(\frac{a}{0.0381 \mathrm{AU} / \mathrm{y}^{2}}\right)\left(\frac{r}{1 \mathrm{AU}}\right)^{2}
$$

where $r$ is the current separation projected onto the sky and $M_{J}$ is the mass of Jupiter. If we translate the measured acceleration to physical units, we find $a_{\alpha}=2.8 \times 10^{-3} \mathrm{AU} / \mathrm{y}^{2}$ and $a_{\delta}=$ $0.5 \times 10^{-3} \mathrm{AU} / \mathrm{y}^{2}$, or the total acceleration $a=2.9 \times 10^{-3} \mathrm{AU} / \mathrm{y}^{2}$. Applied to the stellar companion at a projected distance of $5.5^{\prime \prime}$ (the optically measured separation in 2007), we estimate a mass for GJ $896 \mathrm{~B}$ of $M_{B} \approx 0.4 M_{\odot}$. This mass estimate is in rough agreement with expectations for a star of spectral type M4.5 (Kraus et al. 2011).

We can also apply the acceleration as a limit to estimate planetary mass companions. If we take the acceleration $a$ as an upper limit then we can place a limit on a planetary mass, $M_{p} \lesssim 0.08 M_{J}(r / 1 A U)^{2}$. This acceleration limit applies to companions with a period $\gtrsim 4.9 \mathrm{y}$, or at $r \gtrsim 1.7 \mathrm{AU}$ for a stellar mass of $0.2 M_{\odot}$. For closer orbits, we can set a constraint based on the maximal amplitude of angular displacement. We set this limit by computing the periodogram for the residuals in each coordinate after subtraction of the astrometric plus acceleration model (Figure 7). The amplitude of the periodogram falls well below the 95\%-confidence threshold; we find no evidence for a short-period companion. The maximal power in the periodogram corresponds to an upper limit of 0.4 mas in angular displacement. We are sensitive to orbital periods as short as the characteristic separation between epochs $\sim 140$ days; this limit translates to an inner radius cutoff of $\sim 0.3$ AU. In Figure 8, we combine the acceleration limits and the angular displacement limits. The maximal sensitivity is at $2 \mathrm{AU}$ with an upper limit of $0.15 M_{J}$. Thus, we are sensitive to a planet with approximately 3 Neptune masses at large radii from GJ 896A. Note that this limit on the mass applies strictly to face-on systems; orbits at all inclinations will have a maximum acceleration equal to the face-on case but will have smaller instantaneous values depending on the phase of the orbit.

A caveat to this calculation is that the acceleration we measure may not be a upper limit to the effect of a planetary companion if the companion and GJ 896B produce accelerations that almost cancel each other. The agreement in $a_{\alpha}$ between measured and calculated values suggests that this is not likely; the disagreement in $a_{\delta}$ could be due to a planetary companion acceleration that wholly or partially cancels the binary acceleration. A Jupiter mass companion at 2 AU would 
produce the magnitude of acceleration necessary for the cancellation. This cancellation, however, requires a coincidence that is unlikely to persist over a period of observation of this duration. The agreement in $\mu_{\delta}$ between the Hipparcos and RIPL measurements indicates that such a cancellation must persist over a period of $\gtrsim 16 \mathrm{y}$, which is improbable. Improved optical astrometry of the binary system can determine the contribution of GJ 896B to the motion of GJ 896A.

The rms residuals indicate that the centroid of the stellar emission is confined to a physical region $\lesssim 0.3 R_{\odot}$. This is comparable to or less than the photospheric radius of a late-type star (e.g., Kraus et al. 2011). These residuals occur over a range of source flux densities that vary by a factor of $\sim 60$. We conclude that the magnetic activity associated with GJ 896A is symmetric about the stellar surface and/or confined primarily to the disk of the star.

\section{Conclusions}

We have presented here an analysis of RIPL observations of the star GJ 896A. We measure the parallax to an accuracy of 0.2 mas and the proper motion to an accuracy of 0.01 mas $/ y$. We measure an acceleration term of $0.466 \pm 0.045 \mathrm{mas} / \mathrm{y}^{2}$. This term is significantly less than expected based on the model for the orbit of the GJ 896AB system, suggesting an error in the opticallydetermined orbital parameters. Attributing this acceleration to a planetary mass companion, we demonstrate sensitivity to a planetary companion at $2 \mathrm{AU}$ of mass $0.15 M_{J}$.

The residuals that we obtain from our fitting of 0.25 mas in each coordinate over $4.9 \mathrm{y}$ indicate the feasibility of planet searching and characterization with radio astrometry of nearby low mass stars. These residuals can potentially be improved for this star and others through technical improvements to the data analysis including accounting for the motion of the star during the observing epoch and more exact removal of tropospheric fluctuations. The residuals indicate that astrometric jitter from stellar activity on scales of a stellar radius or larger is not a limiting factor in planet astrometry. Improvements in our analysis of systematic errors from stellar motion and tropospheric phase errors will permit us to explore sub-stellar-radius astrometric accuracy.

Future RIPL papers will explore analysis of additional data from this star, improved analysis techniques, and analysis of other stars in the sample. Upgrades to the recording bandwidth of the VLBA (and GBT) will produce significant improvements in the capability to carry out observing programs of this kind. The improved sensitivity will allow more rapid detection of bright stars such as GJ 896A, thereby reducing any smearing effect from stellar motion, allow the use of closer calibrators for improved tropospheric corrections and reduced time on calibrator, provide higher signal to noise ratio detections of fainter stars, and enable us to detect a larger sample of stars.

The National Radio Astronomy Observatory is a facility of the National Science Foundation operated under cooperative agreement by Associated Universities, Inc. This research has made use of the NASA/IPAC Extragalactic Database (NED) which is operated by the Jet Propulsion 
Laboratory, California Institute of Technology, under contract with the National Aeronautics and Space Administration. This research has made use of the SIMBAD database, operated at CDS, Strasbourg, France. This research was supported by NASA Origins grant NNX07AP19G.

\section{REFERENCES}

Beaulieu, J.-P., Bennett, D. P., Fouqué, P., et al. 2006, Nature, 439, 437

Bennett, D. P., Bond, I. A., Udalski, A., et al. 2008, ApJ, 684, 663

Benz, A. O., Alef, W., \& Guedel, M. 1995, A\&A, 298, 187

Borucki, W. J., Koch, D. G., Basri, G., et al. 2011, ApJ, 728, 117

Boss, A. P. 2006, ApJ, 643, 501

Boss, A. P., Weinberger, A. J., Anglada-Escudé, G., et al. 2009, PASP, 121, 1218

Bower, G. C., Bolatto, A., Ford, E., Kalas, P., \& Ulvestad, J. 2007, ArXiv e-prints

Bower, G. C., Bolatto, A., Ford, E. B., \& Kalas, P. 2009, ApJ, 701, 1922

Brunthaler, A., Reid, M. J., Menten, K. M., et al. 2011, Astronomische Nachrichten, 332, 461

Butler, R. P., Wright, J. T., Marcy, G. W., et al. 2006, ApJ, 646, 505

Endl, M., Cochran, W. D., Kürster, M., et al. 2006, ApJ, 649, 436

Fomalont, E. B., \& Kopeikin, S. M. 2003, ApJ, 598, 704

Forbrich, J., \& Berger, E. 2009, ApJ, 706, L205

Gagne, M., Valenti, J., Johns-Krull, C., et al. 1998, in Astronomical Society of the Pacific Conference Series, Vol. 154, Cool Stars, Stellar Systems, and the Sun, ed. R. A. Donahue \& J. A. Bookbinder, 1484-+

Gaudi, B. S., Bennett, D. P., Udalski, A., et al. 2008, Science, 319, 927

Gould, A., Udalski, A., An, D., et al. 2006, ApJ, 644, L37

Greisen, E. W. 2003, in Astrophysics and Space Science Library, Vol. 285, Astrophysics and Space Science Library, ed. A. Heck, 109

Guirado, J. C., Martí-Vidal, I., Marcaide, J. M., et al. 2006, A\&A, 446, 733

Heintz, W. D. 1984, AJ, 89, 1063

Ida, S., \& Lin, D. N. C. 2005, ApJ, 626, 1045 
Jackson, P. D., Kundu, M. R., \& White, S. M. 1989, A\&A, 210, 284

Johnson, J. A., Butler, R. P., Marcy, G. W., et al. 2007, ApJ, 670, 833

Kennedy, G. M., Kenyon, S. J., \& Bromley, B. C. 2007, Ap\&SS, 311, 9

Kraus, A. L., Tucker, R. A., Thompson, M. I., Craine, E. R., \& Hillenbrand, L. A. 2011, ApJ, 728, 48

Laughlin, G., Bodenheimer, P., \& Adams, F. C. 2004, ApJ, 612, L73

Lestrade, J.-F., Preston, R. A., Jones, D. L., et al. 1999, A\&A, 344, 1014

Loinard, L., Torres, R. M., Mioduszewski, A. J., et al. 2007, ApJ, 671, 546

Mason, B. D., Wycoff, G. L., Hartkopf, W. I., Douglass, G. G., \& Worley, C. E. 2001, AJ, 122, 3466

Menten, K. M., Reid, M. J., Forbrich, J., \& Brunthaler, A. 2007, A\&A, 474, 515

Muterspaugh, M. W., Lane, B. F., Kulkarni, S. R., et al. 2010, AJ, 140, 1657

Pallavicini, R., Willson, R. F., \& Lang, K. R. 1985, A\&A, 149, 95

Sandstrom, K. M., Peek, J. E. G., Bower, G. C., Bolatto, A. D., \& Plambeck, R. L. 2007, ApJ, 667,1161

Torres, R. M., Loinard, L., Mioduszewski, A. J., \& Rodríguez, L. F. 2009, ApJ, 698, 242

van Leeuwen, F. 2007, A\&A, 474, 653

Xu, Y., Moscadelli, L., Reid, M. J., et al. 2011, ApJ, 733, 25

Xu, Y., Reid, M. J., Zheng, X. W., \& Menten, K. M. 2006, Science, 311, 54 


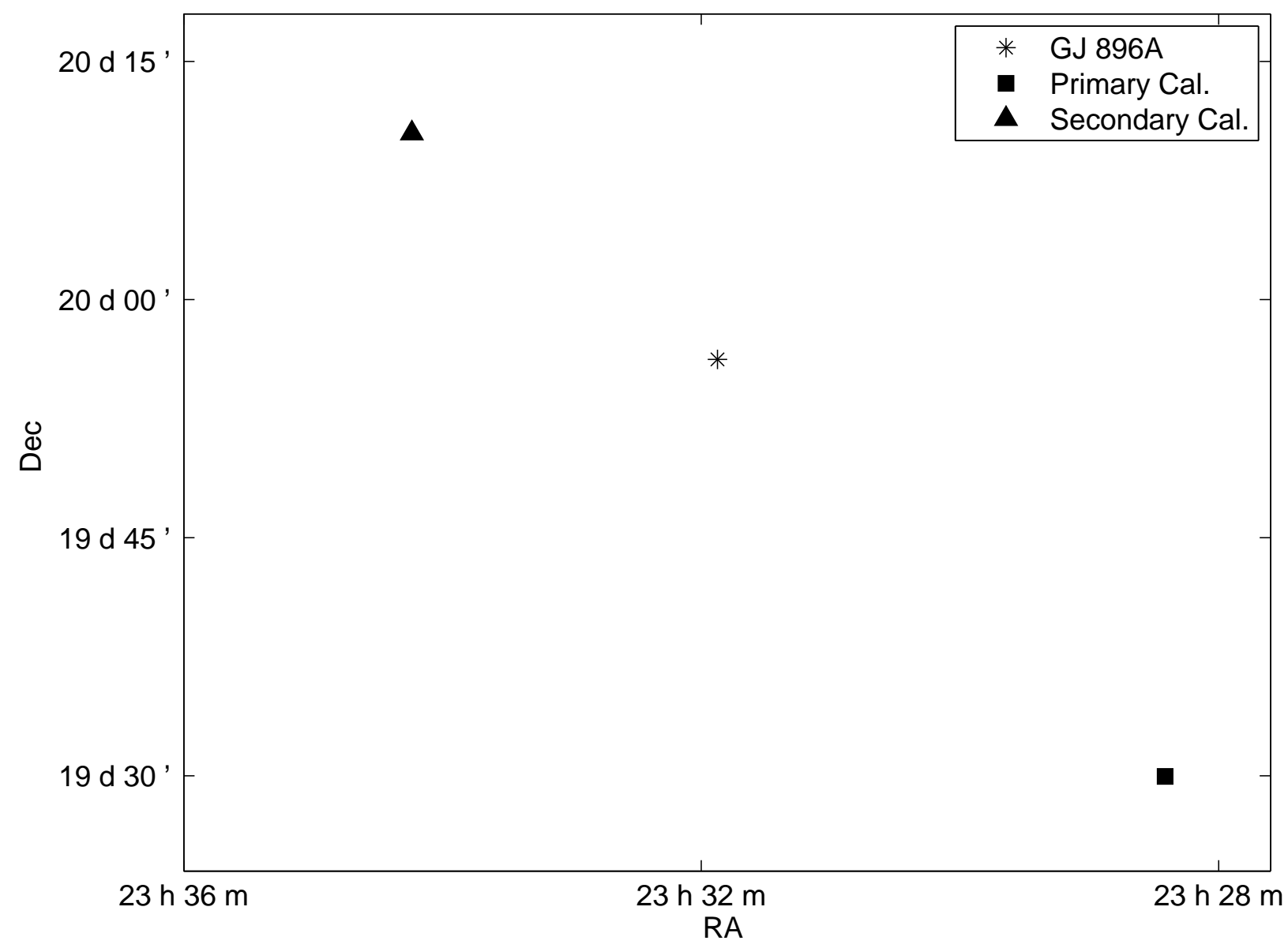

Fig. 1.- Positions for GJ 896A and primary and secondary calibrators. The distance from the primary calibrator, J2328+1929, to GJ 896A is 0.9 deg. The distance from the secondary calibrator, $\mathrm{J} 2334+2010$, to GJ $896 \mathrm{~A}$ is $0.6 \mathrm{deg}$. The nearly linear arrangement and small separations make this an excellent set of calibrators for this star. 

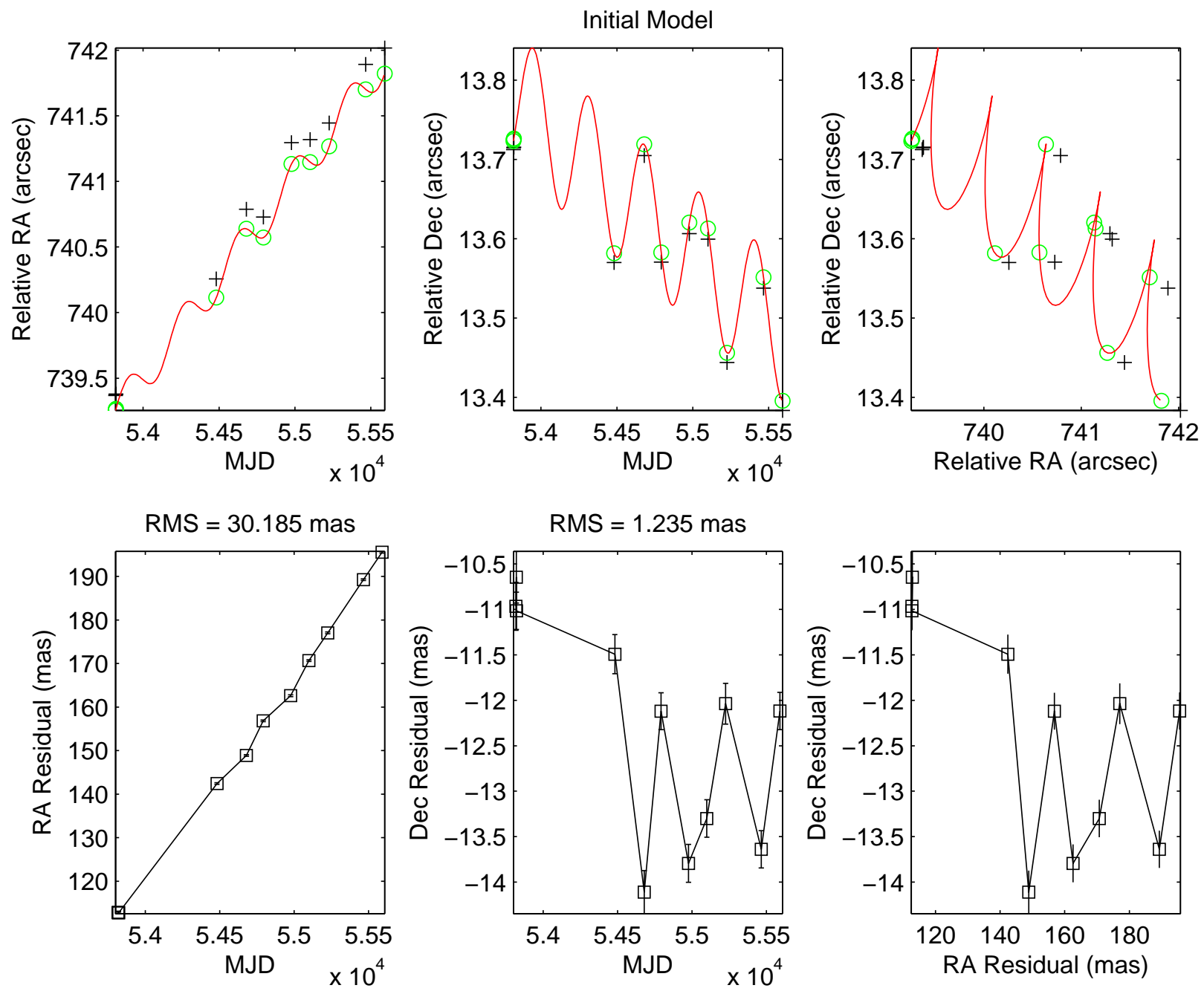

Fig. 2.- Initial model and astrometric data for GJ 896A. Top row shows astrometric data (black crosses) with the initial (optically-determined) astrometric model (green circles at the epoch of observation and red lines for the continuous calculation). Positions in the top row are given in arcsec relative to a fiducial position of $23^{h} 31^{m}, 19^{\circ} 56^{\prime}$. Bottom row shows residuals after subtraction of the model. The symbols in the top row are much larger than the errors; in the bottom row, errors include statistical and systematic contributions. 

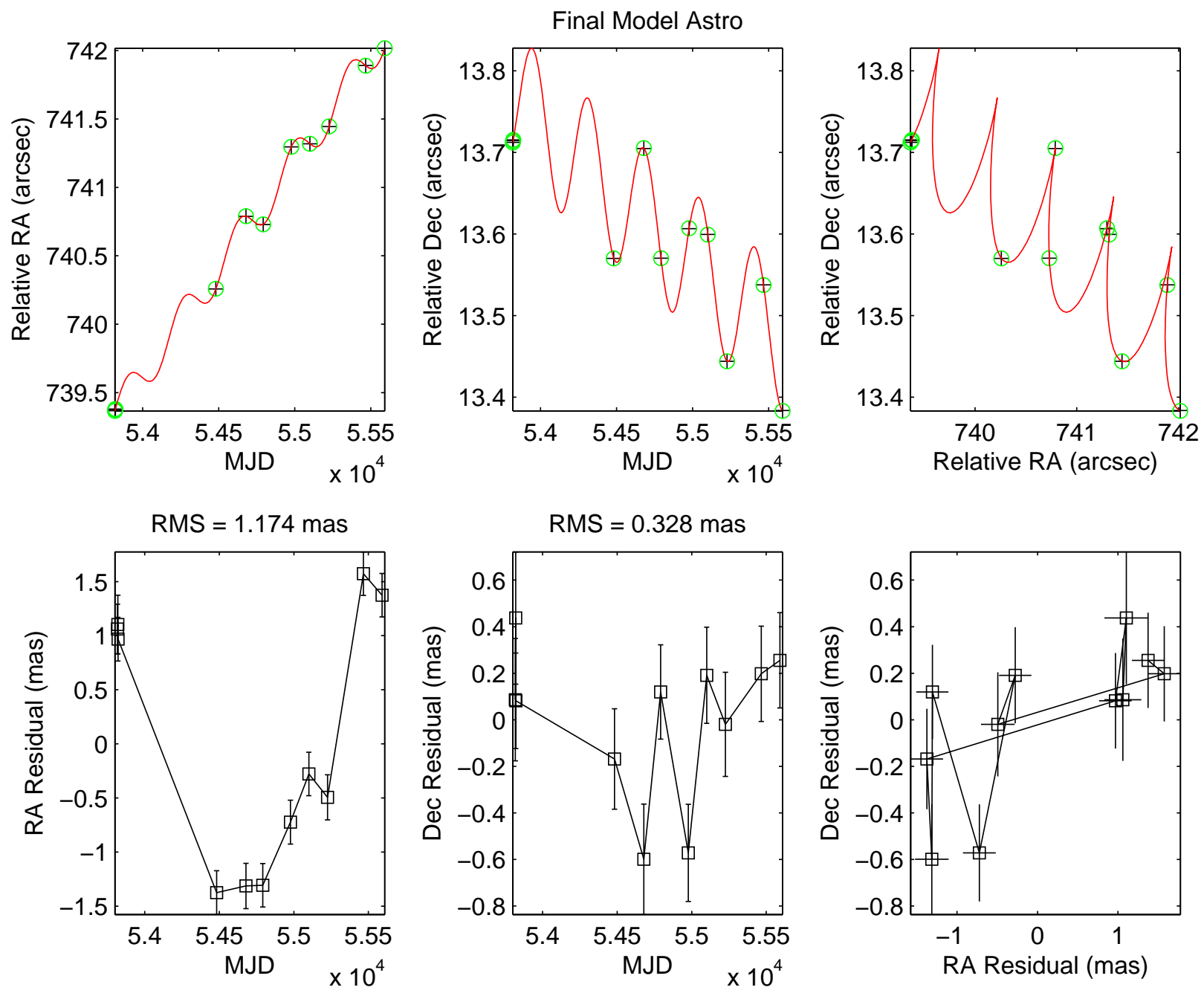

Fig. 3. - Fitted astrometric model and astrometric data for GJ 896A. Symbols are the same as in Figure 2 , 

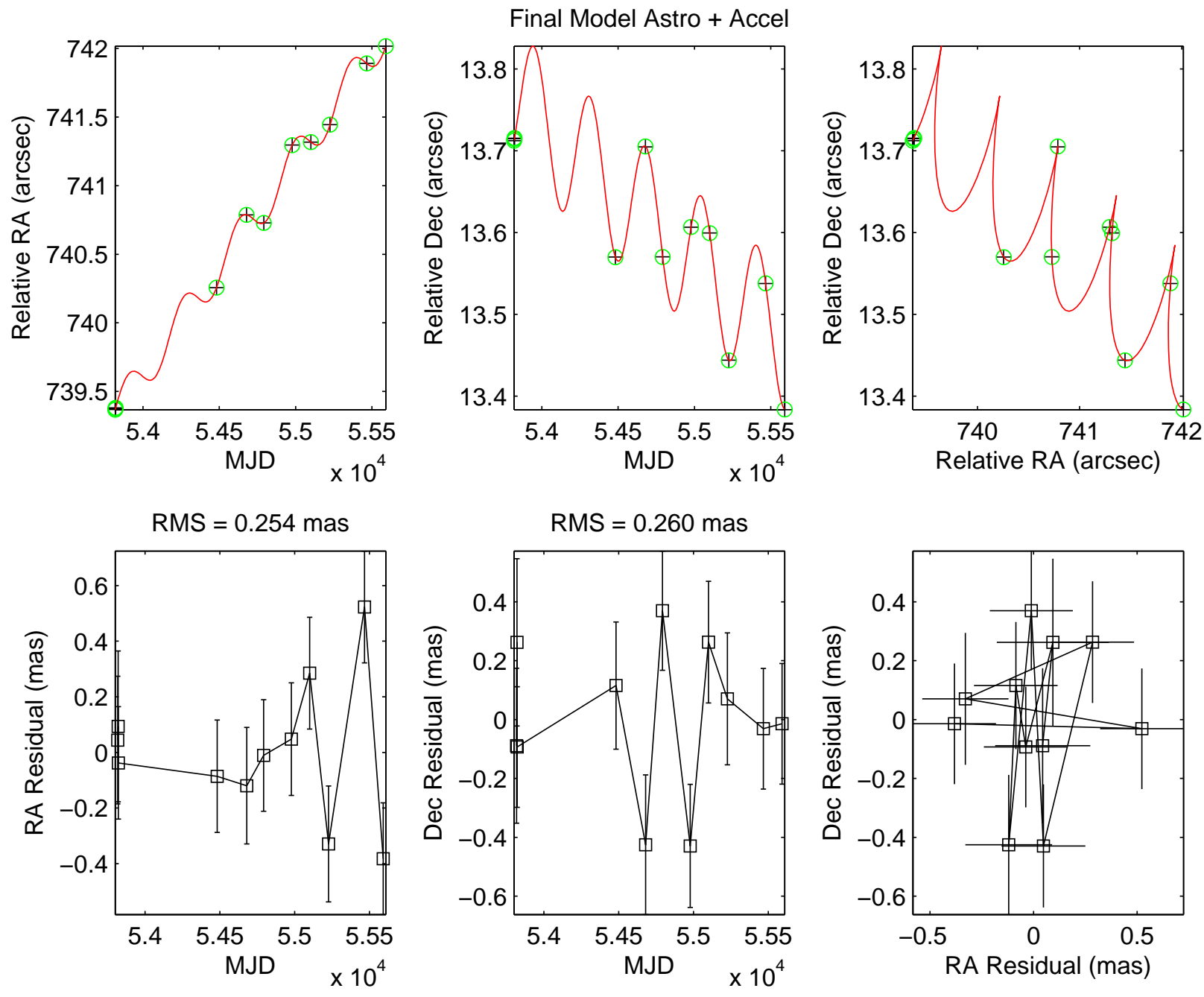

Fig. 4.- Fitted astrometric plus acceleration model and astrometric data for GJ 896A. Symbols are the same as in Figure 2 , 

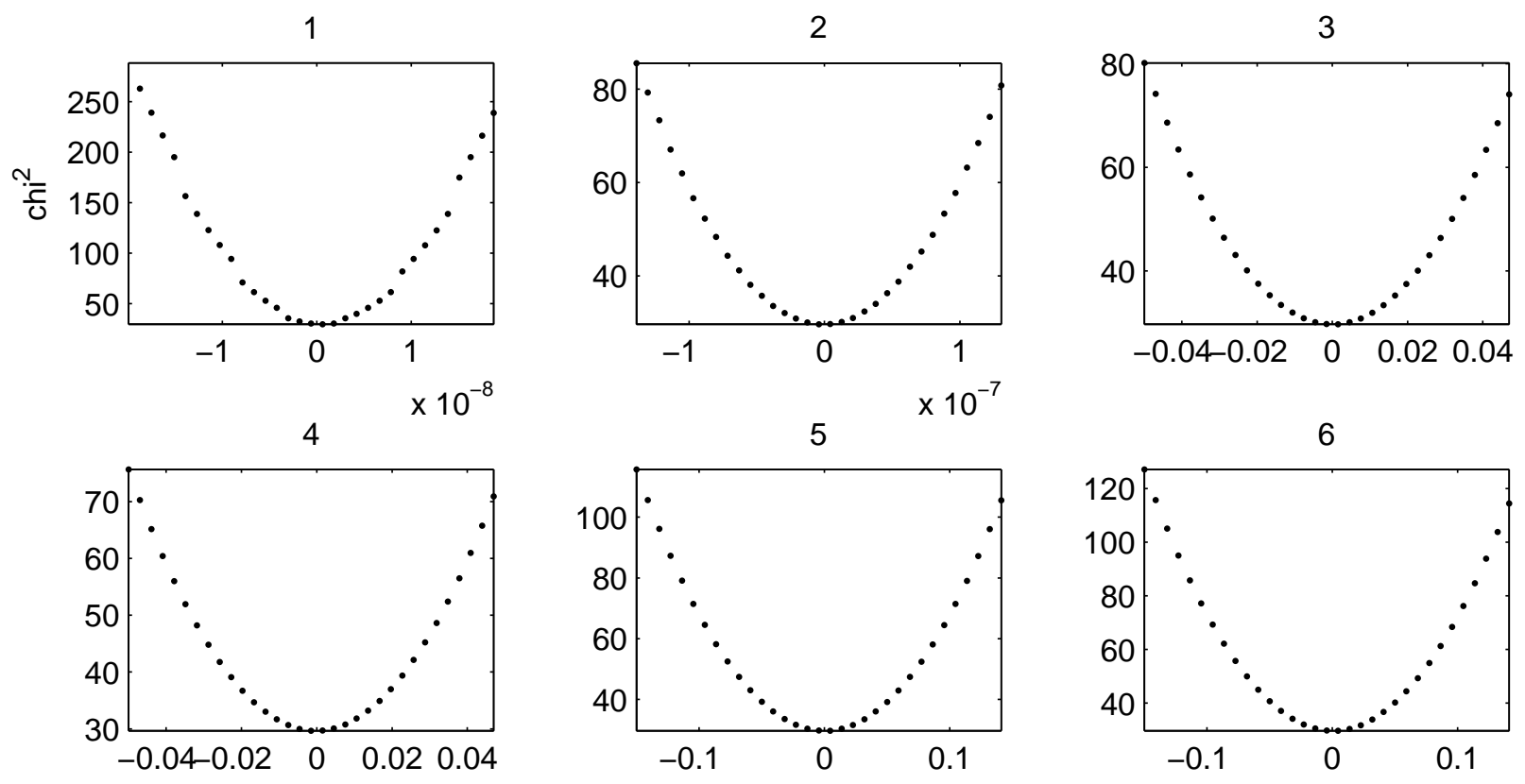

7
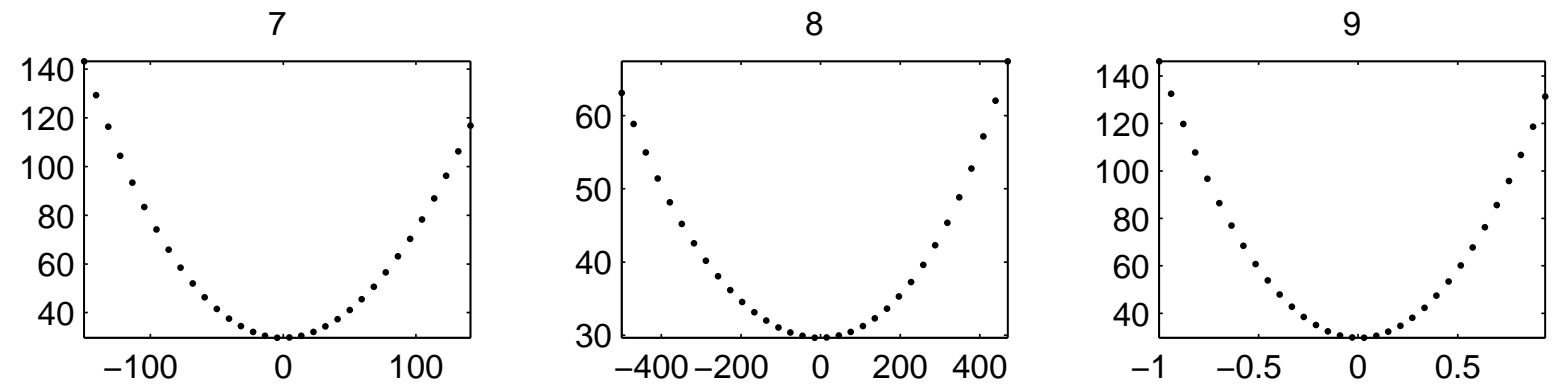

Fig. 5. - $\chi^{2}$ as a function of each parameter. The $\mathrm{x}$-axis values are offsets from the best-fit value. The parameters explored are (1) right ascension (hours), (2) declination (degrees), (3) proper motion in right ascension (mas/y), (4) proper motion in declination (mas/y), (5) acceleration in right ascension $\left(\mathrm{mas} / \mathrm{y}^{2}\right),(6)$ acceleration in declination $\left(\mathrm{mas} / \mathrm{y}^{2}\right),(7)$ minimum time for the right ascension acceleration (days), (8) maximum time for the declination acceleration (days), and (9) parallax (mas). 

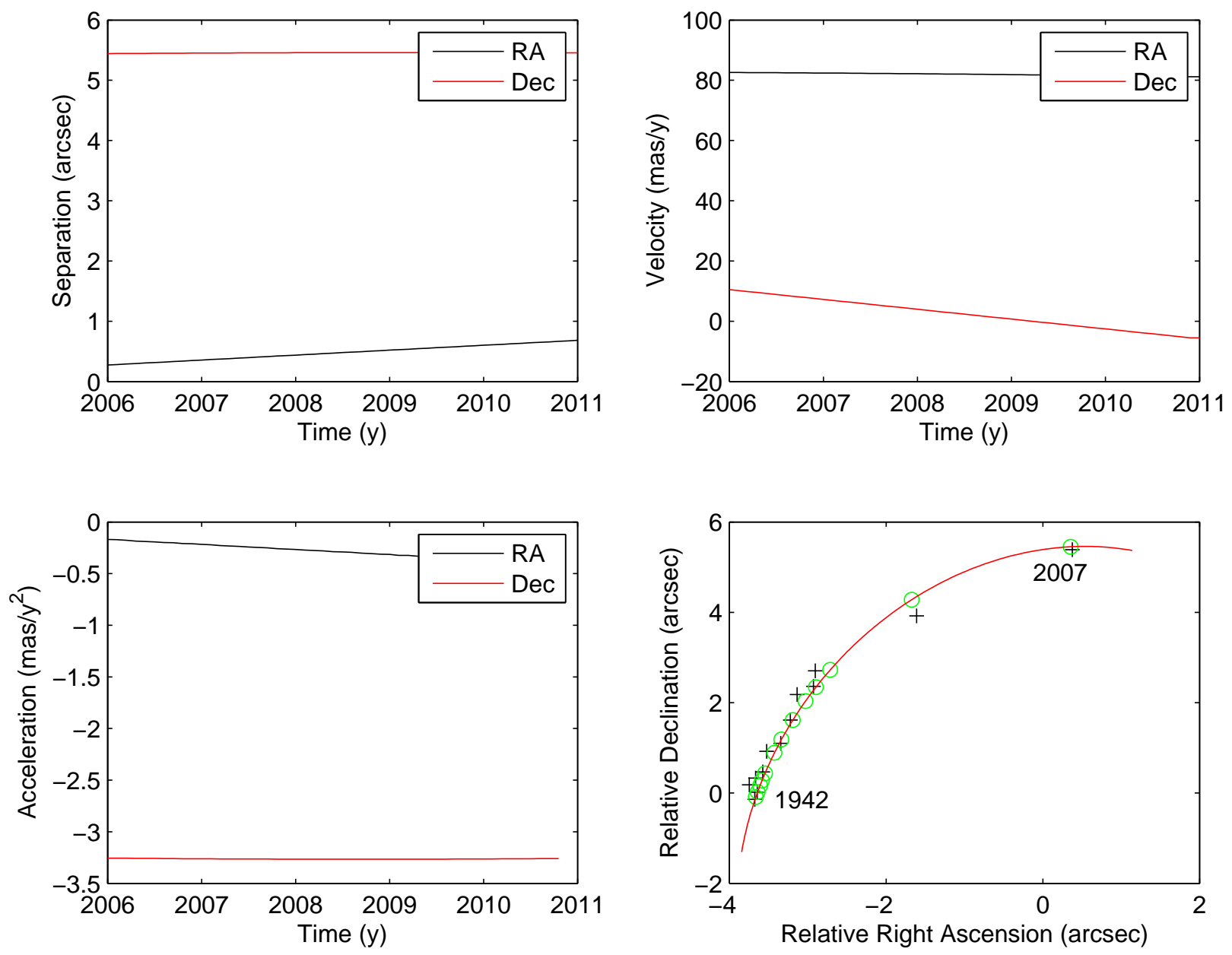

Fig. 6. - Orbital motion for GJ 896B relative to GJ 896A based on optical astrometric data. We plot the relative separation, velocity, and acceleration in the upper left, upper right, and lower left, respectively. We plot in the lower right the historical data (black crosses) from which this orbit derived (Heintz 1984) and the predictions of the orbital model (green circles at the time of measurement, red line for continuous time). We include an additional datum from 2007 that was not part of the fit (Mason et al. 2001). The plotted model curve extends from 1932 to 2017. 


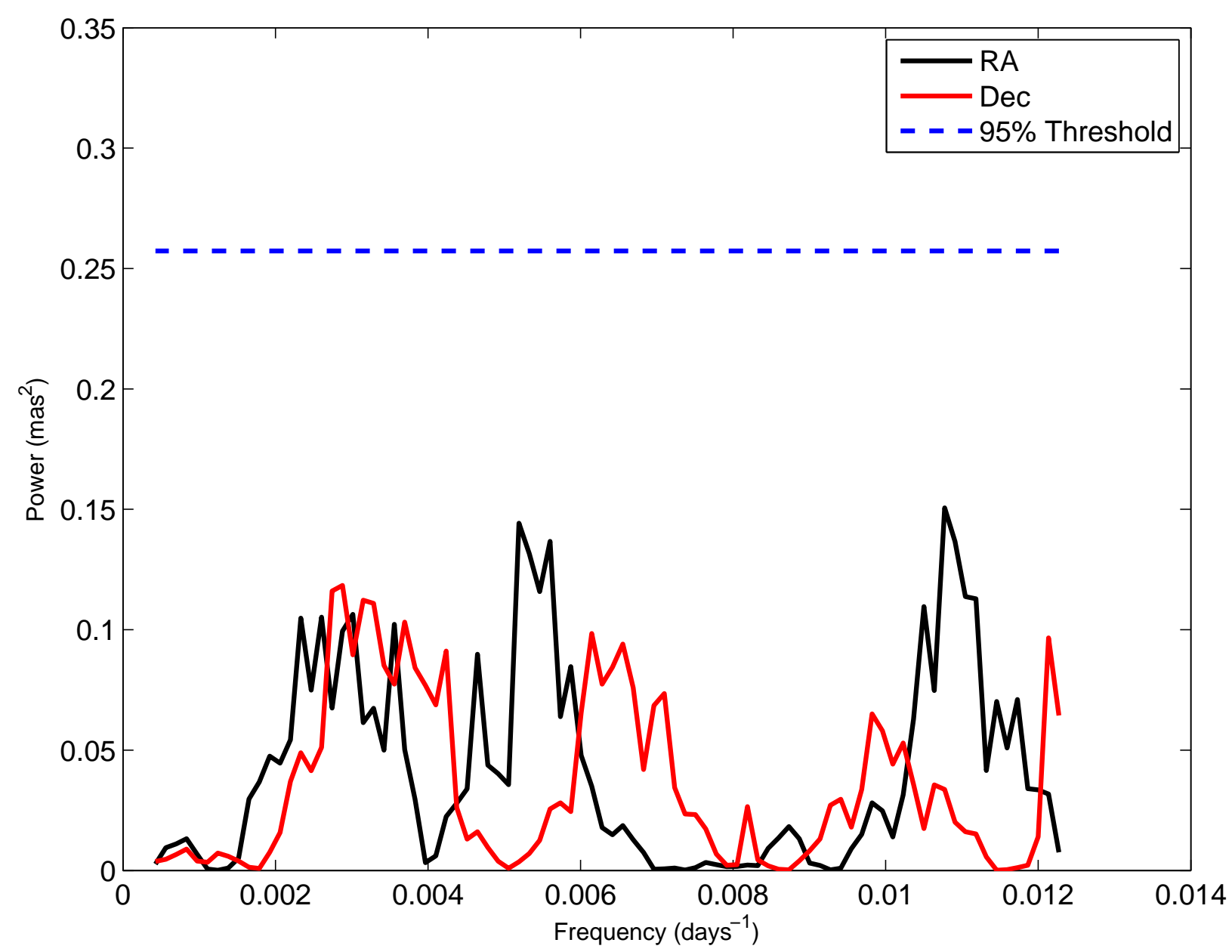

Fig. 7.- Periodogram power as a function of frequency for residuals after removal of astrometric and acceleration terms for the two coordinates. We also plot the $95 \%$ confidence threshold as the blue dashed line. The absence of a significant peak in the periodogram places limits on short-period companions. 


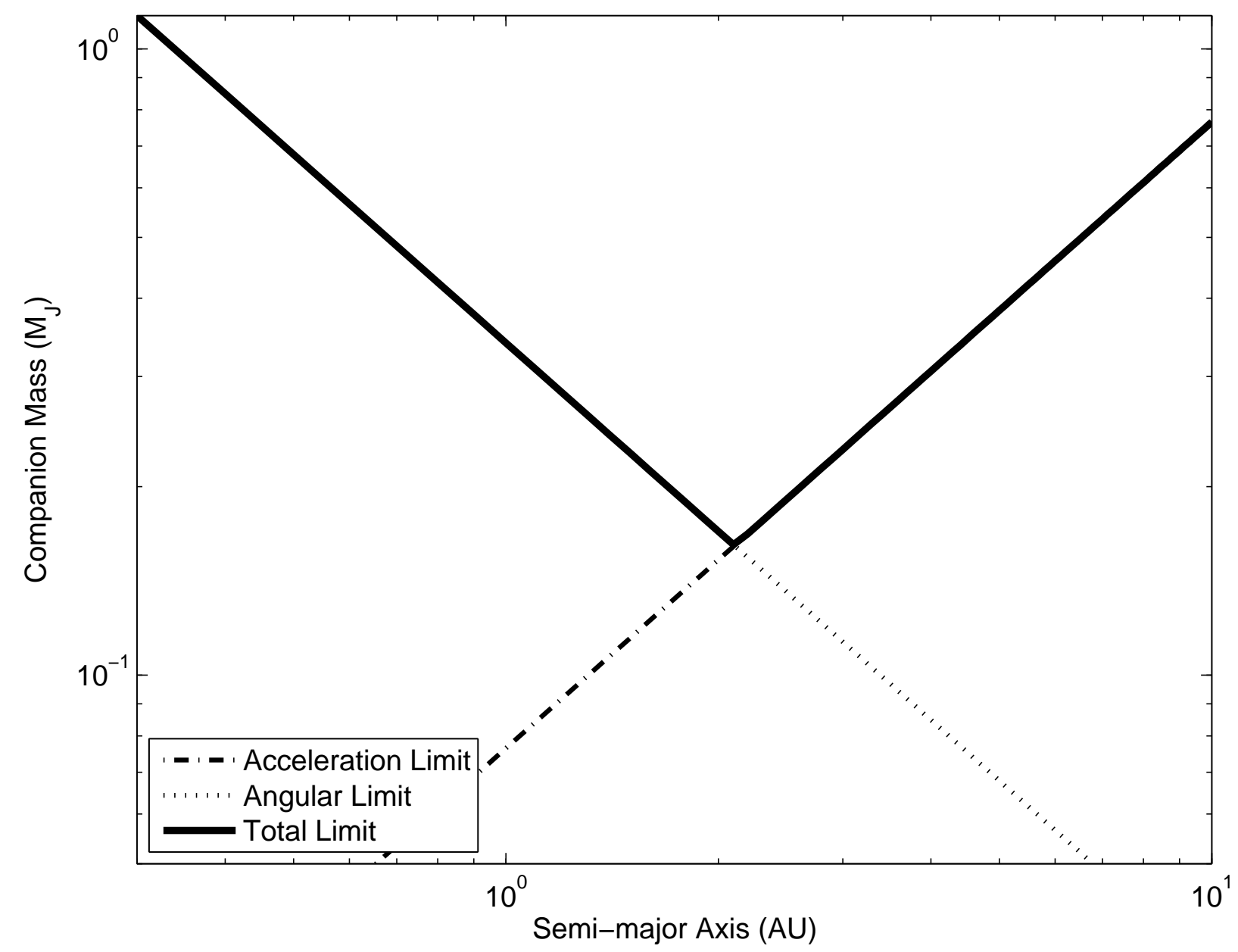

Fig. 8. - Limits on companion mass as a function of semi-major axis for GJ 896A. Limits are derived from acceleration and angular separation terms. The space below the solid curve is allowed. 
Table 1. Positions for Secondary Calibrator J2334+2010

\begin{tabular}{cccccc}
\hline \hline JD & $\begin{array}{c}\text { Flux } \\
(\mathrm{mJy})\end{array}$ & $\begin{array}{c}\alpha \\
(\mathrm{J} 2000)\end{array}$ & $\begin{array}{c}\Delta \alpha \\
(\mathrm{mas})\end{array}$ & $\begin{array}{c}\delta \\
(\mathrm{J} 2000)\end{array}$ & $\begin{array}{c}\Delta \delta \\
(\mathrm{mas})\end{array}$ \\
\hline 2453820 & $62.2 \pm 1.9$ & 233414.1564960 & 0.135 & 201028.882640 & 0.279 \\
\hline 2454482 & $24.7 \pm 0.2$ & 233414.1564897 & 0.007 & 201028.882867 & 0.013 \\
2454585 & $19.6 \pm 0.3$ & 233414.1564998 & 0.013 & 201028.882864 & 0.022 \\
2454680 & $13.1 \pm 0.3$ & 233414.1564977 & 0.018 & 201028.882824 & 0.033 \\
2454793 & $15.9 \pm 0.3$ & 233414.1565001 & 0.015 & 201028.882793 & 0.025 \\
2454978 & $21.4 \pm 0.3$ & 233414.1564972 & 0.010 & 201028.882838 & 0.017 \\
2455101 & $23.6 \pm 0.2$ & 233414.1564926 & 0.007 & 201028.883053 & 0.015 \\
2455226 & $19.0 \pm 0.1$ & 233414.1565001 & 0.004 & 201028.882733 & 0.006 \\
2455467 & $23.8 \pm 0.2$ & 233414.1565155 & 0.006 & 201028.882853 & 0.010 \\
2455591 & $21.8 \pm 0.2$ & 233414.1565109 & 0.006 & 201028.882888 & 0.010 \\
\hline
\end{tabular}

Note. - The first line is the mean position reported in Paper I. Errors in the position are errors from fitting and do not include systematic effects. 
Table 2. Positions for GJ 896A

\begin{tabular}{crcccc}
\hline \hline JD & \multicolumn{1}{c}{$\begin{array}{l}\text { Flux } \\
(\mu \mathrm{Jy})\end{array}$} & $\begin{array}{c}\alpha \\
(\mathrm{J} 2000)\end{array}$ & $\begin{array}{c}\Delta \alpha \\
(\mathrm{mas})\end{array}$ & $\begin{array}{c}\delta \\
(\mathrm{J} 2000)\end{array}$ & $\begin{array}{c}\Delta \delta \\
(\mathrm{mas})\end{array}$ \\
\hline & & & & & \\
\hline 2453818.310 & $929 \pm 203$ & 233152.4336850 & 0.113 & 195613.712351 & 0.171 \\
2453820.310 & $1399 \pm 298$ & 233152.4342630 & 0.183 & 195613.714736 & 0.202 \\
2453821.310 & $3895 \pm 230$ & 233152.4345400 & 0.028 & 195613.715419 & 0.045 \\
\hline 2454482.443 & $328 \pm 20$ & 233152.4967356 & 0.031 & 195613.570094 & 0.081 \\
2454679.922 & $278 \pm 25$ & 233152.5343729 & 0.062 & 195613.704882 & 0.128 \\
2454792.651 & $2041 \pm 52$ & 233152.5301598 & 0.015 & 195613.570517 & 0.031 \\
2454978.109 & $9512 \pm 470$ & 233152.5703944 & 0.033 & 195613.606525 & 0.060 \\
2455100.776 & $1139 \pm 33$ & 233152.5719845 & 0.018 & 195613.599548 & 0.051 \\
2455226.443 & $138 \pm 16$ & 233152.5809398 & 0.059 & 195613.443975 & 0.101 \\
2455466.776 & $2484 \pm 88$ & 233152.6126099 & 0.021 & 195613.537846 & 0.045 \\
2455591.443 & $2018 \pm 78$ & 233152.6215506 & 0.018 & 195613.383628 & 0.044 \\
\hline
\end{tabular}

Note. - The first three measurements are the results from Paper I.

Table 3. Astrometric Parameters for GJ 896A

\begin{tabular}{lrrrr}
\hline \hline Parameter & \multicolumn{1}{c}{ Unit } & \multicolumn{1}{c}{ Optical } & RIPL Astrometric & RIPL Astrometric + Accel \\
\hline$\alpha_{0}$ & & 233152.17898 & 233152.179555 & 233152.179565 \\
$\Delta \alpha_{0}$ & $(\mathrm{mas})$ & 12.31 & 0.409 & 0.123 \\
$\delta_{0}$ & & 195614.1505 & 195614.141024 & 195614.139842 \\
$\Delta \delta_{0}$ & $(\mathrm{mas})$ & 9.51 & 0.434 & 0.136 \\
$\mu_{\alpha}$ & $(\mathrm{mas} / \mathrm{y})$ & $554.64 \pm 1.40$ & $571.333 \pm 0.045$ & $571.169 \pm 0.014$ \\
$\mu_{\delta}$ & $(\mathrm{mas} / \mathrm{y})$ & $-60.43 \pm 1.08$ & $-60.781 \pm 0.047$ & $-60.676 \pm 0.014$ \\
$\pi$ & $(\mathrm{mas})$ & $161.76 \pm 1.66$ & $159.864 \pm 0.616$ & $160.010 \pm 0.182$ \\
$a_{\alpha}$ & $\left(\mathrm{mas} / \mathrm{y}^{2}\right)$ & $\ldots$ & $\ldots$ & $0.458 \pm 0.032$ \\
$a_{\delta}$ & $\left(\mathrm{mas} / \mathrm{y}^{2}\right)$ & $\ldots$ & $\ldots$ & $0.087 \pm 0.032$ \\
$t_{0}^{\alpha}$ & $(\mathrm{JD})$ & $\ldots$ & $\ldots$ & $2454565 \pm 32$ \\
$t_{0}^{\delta}$ & $(\mathrm{JD})$ & & & $2454879 \pm 167$ \\
\hline
\end{tabular}


Table 4. Orbital Parameters for the GJ 896 AB System From Optical Astrometry

\begin{tabular}{crrrrrr}
\hline \hline $\begin{array}{c}\text { Period } \\
(\mathrm{y})\end{array}$ & $\begin{array}{c}\text { Semi-Major Axis } \\
(\operatorname{arcsec})\end{array}$ & $\begin{array}{c}\text { Inclination } \\
(\mathrm{deg})\end{array}$ & $\begin{array}{c}\Omega \\
(\mathrm{deg})\end{array}$ & $\begin{array}{c}\text { Periastron } \\
(\mathrm{y})\end{array}$ & Eccentricity & $\begin{array}{c}\omega \\
(\mathrm{deg})\end{array}$ \\
\hline 359 & 6.87 & 123.5 & 82.1 & 2008 & 0.20 & 354.0 \\
\hline
\end{tabular}

